\title{
Effects of polymer molecular weight on relative oral bioavailability of curcumin
}

\author{
This article was published in the following Dove Press journal: \\ International Journal of Nanomedicine \\ 14 June 2012 \\ Number of times this article has been viewed
}

\author{
Yin-Meng Tsai' \\ Wan-Ling Chang-Liao' \\ Chao-Feng Chien' \\ Lie-Chwen Lin ${ }^{1,2}$ \\ Tung-Hu Tsai ${ }^{1,3}$ \\ 'Institute of Traditional Medicine, \\ School of Medicine, National \\ Yang-Ming University, ${ }^{2} \mathrm{National}$ \\ Research Institute of Chinese \\ Medicine, ${ }^{3}$ Department of Education \\ and Research, Taipei City Hospital, \\ Taipei, Taiwan
}

Background: Polylactic-co-glycolic acid (PLGA) nanoparticles have been used to increase the relative oral bioavailability of hydrophobic compounds and polyphenols in recent years, but the effects of the molecular weight of PLGA on bioavailability are still unknown. This study investigated the influence of polymer molecular weight on the relative oral bioavailability of curcumin, and explored the possible mechanism accounting for the outcome.

Methods: Curcumin encapsulated in low (5000-15,000) and high $(40,000-75,000)$ molecular weight PLGA (LMw-NPC and HMw-NPC, respectively) were prepared using an emulsificationsolvent evaporation method. Curcumin alone and in the nanoformulations was administered orally to freely mobile rats, and blood samples were collected to evaluate the bioavailability of curcumin, LMw-NPC, and HMw-NPC. An ex vivo experimental gut absorption model was used to investigate the effects of different molecular weights of PLGA formulation on absorption of curcumin. High-performance liquid chromatography with diode array detection was used for quantification of curcumin in biosamples.

Results: There were no significant differences in particle properties between LMw-NPC and HMw-NPC, but the relative bioavailability of HMw-NPC was 1.67-fold and 40-fold higher than that of LMw-NPC and conventional curcumin, respectively. In addition, the mean peak concentration $\left(\mathrm{C}_{\max }\right)$ of conventional curcumin, LMw-NPC, and HMw-NPC was 0.028, 0.042, and $0.057 \mu \mathrm{g} / \mathrm{mL}$, respectively. The gut absorption study further revealed that the HMw-PLGA formulation markedly increased the absorption rate of curcumin in the duodenum and resulted in excellent bioavailability compared with conventional curcumin and LMw-NPC.

Conclusion: Our findings demonstrate that different molecular weights of PLGA have varying bioavailability, contributing to changes in the absorption rate at the duodenum. The results of this study provide the rationale for design of a nanomedicine delivery system to enhance the bioavailability of water-insoluble pharmaceutical compounds and functional foods.

Keywords: absorption, duodenum, molecular weight, poly(lactic-co-glycolic acid), PLGA, relative oral bioavailability

\section{Introduction}

Polyphenols exist widely in plants and plant-derived foods, including vegetables, fruit, tea, spices, wine, beverages, and nutritional supplement products, thus constitute an important part of the human diet. Previous studies have demonstrated that polyphenols have broad pharmacological activity and biological benefits, including anticancer, neuroprotection, chemoprevention, and protection against cardiovascular disease. ${ }^{1}$ These advantages are attributed to their useful antioxidant and anti-inflammatory properties that regulate cell proliferation and function to prevent onset and progression of various human diseases. ${ }^{2}$ However, the beneficial effects of many polyphenols on human
Correspondence: Tung-Hu Tsai National Yang-Ming University,

School of Medicine, Institute

of Traditional Medicine,

Taipei I 12, Taiwan

Tel +88 6228267115

Fax +88 6228225044

Email thtsai@ym.edu.tw 
health are limited due to their low oral bioavailability. This is because the polyphenols, including curcumin, ${ }^{3}$ resveratrol, ${ }^{4}$ quercetin, ${ }^{5}$ daidzein, ${ }^{6}$ silymarin, ${ }^{7}$ and other polyphenols ${ }^{2}$ are poorly absorbed in the gut and undergo fast metabolism by the liver.

To improve the low oral bioavailability of polyphenols, researchers have investigated their encapsulation in different nanoformulation systems, including micelles, liposomes, and nanoparticles. ${ }^{2,8}$ The mechanism by which these nanoformulations enhance the oral bioavailability of polyphenols involves increasing the surface area and interaction of the compounds, thereby raising the dissolution rate to improve their absorption. ${ }^{9}$ Nevertheless, previous studies have shown that polyester nanoparticle-based formulations are suitable for oral ingestion because they are more stable than other formulations in the gastrointestinal tract. ${ }^{10}$ Polyesters, for instance, polylactic-co-glycolic acid (PLGA), are the substances most often used in nanoformulations and have been approved by the US Food and Drug Administration as therapeutic devices owing to their biocompatibility, biodegradability, and versatile degradation kinetics. ${ }^{11}$ Recent research has shown that nanoformulations based on PLGA and nanogels have lower human serum protein binding and are more compatible than $\beta$-cyclodextrin, cellulose, and dendrimers with human erythrocytes. ${ }^{12}$ Accordingly, PLGA nanoparticle systems have been used extensively to increase the oral bioavailability of polyphenols. Sonaje et al have demonstrated that the oral bioavailability of ellagic acid is increased when it is nanoformulated with PLGA. ${ }^{13}$ Another study has shown that quercetin encapsulated in PLGA nanoparticles has an increased ability to protect the liver and brain against oxidative damage. ${ }^{14}$ Curcumin in a PLGA nanoformulation not only has increased oral bioavailability but is also taken up in increased amounts by metastatic cancer cells. ${ }^{3,15}$ Xie et al have reported that the improved oral bioavailability of curcumin when encapsulated in PLGA nanoparticles may be attributable to enhanced water solubility, improved permeability, and inhibition of P-glycoprotein-mediated efflux in the intestine. ${ }^{16}$

Although PLGA nanoformulations are effective in improving the oral bioavailability of polyphenols, many kinds of PLGA with different molecular weights are available on the market. Our knowledge about the influence of the molecular weight of PLGA on the oral bioavailability of polyphenols is limited. Hence, the aim of this work was to investigate the effects of the molecular weight of PLGA on the oral bioavailability of polyphenols. Curcumin, one of the biologically active polyphenols, is a candidate compound for further pharmacokinetic study.

\section{Materials and methods}

\section{Chemicals and reagents}

Polyvinyl alcohol (molecular weight 9000-10,000), low molecular weight PLGA (50:50, molecular weight 5000-15,000), high molecular weight PLGA (50:50, molecular weight 40,000-75,000), monosodium phosphate, sucrose, 2-(4'-hydroxybenzeneazo)benzoic acid (as the internal standard), and polyethylene glycol 400 were obtained from Sigma-Aldrich (St Louis, MO). Curcumin (purity $\geq 95 \%$ ) was purchased from Fluka (Buchs, Switzerland). Acetonitrile and dichloromethane were obtained from Merck (Darmstadt, Germany). Milli-Q grade water (Millipore, Bedford, MA) was used for preparation of the mobile phase and solution.

\section{Encapsulation of curcumin in PLGA nanoparticles}

A high-pressure emulsification-solvent evaporation technique was used to encapsulate curcumin in PLGA nanoparticles, as reported previously. ${ }^{17}$ In brief, $50 \mathrm{mg}$ of PLGA (low or high molecular weight) and $5 \mathrm{mg}$ of curcumin were added to $1.25 \mathrm{~mL}$ of dichloromethane as the oil phase and sonicated for 5 minutes to dissolve all substances. This oil phase was added to $10 \mathrm{~mL}$ of aqueous phase (containing $2 \%$ polyvinyl alcohol and $20 \%$ sucrose, w/v) and then homogenized using a Polytron PT-MR 2100 (Kinematica AG, Lucerne, Switzerland) at 28,000 rpm for 10 minutes to form an emulsion. The emulsion was passed across a $0.1 \mu \mathrm{m}$ filter twice at an operating pressure of $5 \mathrm{~kg} / \mathrm{cm}^{2}$ using an accessory extruder (EF-C5, Avestin, Canada) to formulate low and high molecular weight PLGA nanoparticles encapsulating curcumin (LMw-NPC and HMw-NPC, respectively). The resulting nanoparticles were stirred at $500 \mathrm{rpm}$ overnight and air-dried to evaporate the organic solvent.

\section{Characteristics of LMw-NPC and $\mathrm{HMw}-\mathrm{NPC}$}

The particle size and polydispersity index of the LMw-NPC and $\mathrm{HMw}-\mathrm{NPC}$ were measured by dynamic light scattering (90Plus, BIC, Holtsville, NY). The zeta potential of the LMw-NPC and HMw-NPC was determined using a zeta potential analyzer (90Plus, BIC).

\section{Entrapment efficiency}

To evaluate the entrapment efficiency of curcumin encapsulated in PLGA nanoparticles, LMw-NPC and HMw-NPC solutions were centrifuged at 11,000 rpm for 15 minutes (Centrifuge 5415R, Eppendorf, Germany). 
After centrifugation, the supernatant was removed and $1 \mathrm{~mL}$ of acetonitrile was then added to the nanoparticle pellet to destroy its structure and allow release of curcumin. The amount of curcumin in the nanoparticles was analyzed by high-performance liquid chromatography (HPLC). Percent entrapment efficiency is given by:

$$
\left(\text { Curcumin }_{\text {encapsulated }} / \text { Curcumin }_{\text {total }}\right) \times 100
$$

\section{Transmission electron microscopy}

Briefly, the LMw-NPC and HMw-NPC solutions were placed dropwise onto a 400 mesh copper grid coated with carbon. About 15 minutes after nanoparticle deposition, the grid was patted with filter paper to remove surface water and then stained using a solution of phosphotungstic acid $(2 \%, \mathrm{w} / \mathrm{v})$ for 20 minutes. TEM samples were obtained after the stained sample had been allowed to dry in air. Micrographs of LMw-NPC and $\mathrm{HMw}-\mathrm{NPC}$ were obtained using a transmission electron microscope (TEM, JEM-2000EXII, JEOL, Tokyo, Japan).

\section{HPLC system and analytical method validation HPLC system}

The HPLC system comprised a chromatographic pump (LC-20AT, Shimadzu, Kyoto, Japan), autosampler (SIL-20AT, Shimadzu), diode array detector (SPD-M20A, Shimadzu), and degasser (DG-240). A reversed-phase C18 column $(4.6 \times 150 \mathrm{~mm}$, particle size $5 \mu \mathrm{m}$, Eclipse XDB, Agilent, Palo Alto, CA) was used for the HPLC separation. The mobile phase consisted of acetonitrile, $10 \mathrm{mM}$ monosodium phosphate ( $\mathrm{pH} 3.5$ adjusted by phosphoric acid, $40: 60, \mathrm{v} / \mathrm{v}$ ) at a flow rate of $0.8 \mathrm{~mL}$ per minute. The HPLC run time was 27 minutes and the detection wavelength was set at $425 \mathrm{~nm}$. The mobile phase was passed through a $0.45 \mu \mathrm{m}$ Millipore membrane filter and then degassed by sonication 2510R-DTH (Bransonic, CT) before use.

\section{Validation of analytical method}

A stock solution of curcumin was diluted in acetonitrile $500 \mu \mathrm{g}$ / $\mathrm{mL}$ to make serial concentrations of a working standard solution $(0.1,0.25,1,2.5,10$, and $25 \mu \mathrm{g} / \mathrm{mL})$ with $50 \%$ acetonitrile. Calibration standards were prepared using $5 \mu \mathrm{L}$ of the working standard solution spiked with $45 \mu \mathrm{L}$ of blank plasma, feces, and urine. The sample extracted procedures described follow the sample preparation section, on the next page. The calibration curves were represented by the peak area ratio of curcumin to the internal standard spiked in blank matrix as the y axis and the concentration of curcumin as the $\mathrm{x}$ axis.
The limits of detection and quantification were defined as a signal-to-noise ratio of 3 and the lowest concentration of the linear regression, respectively. The accuracy and precision of intraday (same day) and interday (six sequential days) sampling of curcumin were assayed (six individual tests). Accuracy (\% bias) was calculated as $\left[\left(\mathrm{C}_{\mathrm{obs}}-\mathrm{C}_{\text {nom }}\right) / \mathrm{C}_{\mathrm{nom}}\right] \times 100$, while $\mathrm{C}_{\text {nom }}$ represented the nominal concentration and $\mathrm{C}_{\mathrm{obs}}$ indicated the mean value of the observed concentration. Precision was calculated as the relative standard deviation (RSD) from the observed concentrations as follows:

$$
\% \mathrm{RSD}=\left[\text { standard deviation } / \mathrm{C}_{\mathrm{obs}}\right] \times 100 .
$$

The \% bias and \% RSD value for the lowest acceptable reproducibility concentrations were defined as being within $\pm 15 \%$. Extraction recovery (\%) of curcumin was assessed by comparing the peak area ratio of curcumin and the internal standard spiked with blank matrix three times (at concentrations of $0.1,0.25$, and $2.5 \mu \mathrm{g} / \mathrm{mL}$ ) to reference the curcumin prepared in $50 \%$ acetonitrile at the same concentrations.

\section{Animal study}

Male Sprague-Dawley rats $(215 \pm 10 \mathrm{~g}$ body weight $)$ were obtained from the Laboratory Animal Center at National Yang-Ming University (Taipei, Taiwan). The animals were pathogen-free and allowed to adapt to an environmentally controlled habitat $\left(24^{\circ} \mathrm{C} \pm 1^{\circ} \mathrm{C}\right.$ on a 12 -hour light-dark cycle). Food (Laboratory Rodent Diet 5001, PMI Feeds Inc, Richmond, IN) and water were available ad libitum. All animal experiments were performed according to the National Yang-Ming University guidelines, principles, and procedures for the care and use of laboratory animals.

The rats were anesthetized using pentobarbital $50 \mathrm{mg} / \mathrm{kg}$ intraperitoneally. During anesthesia, the right jugular vein was catheterized with polyethylene tubing for blood sampling. An external cannula was fastened in the dorsal neck area. The rats were returned to consciousness after one day and could move freely. Curcumin and the LMw-NPC/HMw-NPC nanoformulations were administered by gavage to the rats at doses of $1 \mathrm{~g} / \mathrm{kg}$ and $50 \mathrm{mg} / \mathrm{kg}$, respectively. After oral administration, a $300 \mu \mathrm{L}$ blood sample was obtained from the right jugular vein into a tube rinsed with heparin at 15 , $30,45,60,90,120,150,180,240,300,360$, and 480 minutes. Fecal and urine samples were collected in metabolic cages (Mini Mitter, Bend, OR) at 0-12, 12-24, 24-36, 36-48, and 48-72 hours after treatment with curcumin, LMw-NPC, or HMw-NPC. 


\section{Sample preparation}

Blood samples were centrifuged at $8000 \mathrm{rpm}$ for 10 minutes at $4^{\circ} \mathrm{C}$ for preparation of the plasma samples. The supernatant was collected and preserved at $-20^{\circ} \mathrm{C}$ before analysis. The feces were air-dried, weighed, and then homogenized in five-fold of their volume $(1 \mathrm{~g}$ feces with $5 \mathrm{ml})$ with $50 \%$ aqueous acetonitrile using the Polytron PT-MR 2100 homogenizer. The fecal samples were then centrifuged at $6000 \mathrm{rpm}$ for 10 minutes at $4^{\circ} \mathrm{C}$, and the supernatant was then collected and preserved at $-20^{\circ} \mathrm{C}$ for further assay. Plasma and fecal samples $(50 \mu \mathrm{L}$, vortex-mixed with $100 \mu \mathrm{L}$ of internal standard solution containing $1.5 \mu \mathrm{g} / \mathrm{mL}$ of 2-(4'-hydroxybenzeneazo)benzoic acid dissolved in acetonitrile) were subjected to protein precipitation. After centrifugation at $12,000 \mathrm{rpm}$ for 15 minutes, $20 \mu \mathrm{L}$ of supernatant was collected and analyzed by HPLC.

The original volume of urine was recorded before centrifugation at $8000 \mathrm{rpm}$ for 10 minutes and collection of the supernatant. Next, $50 \mu \mathrm{L}$ of the urine supernatant was extracted using $1 \mathrm{~mL}$ of ethyl acetate and vortexed for one minute. The ethyl acetate phase was collected by centrifugation (13,000 rpm for one minute), and then transferred to a clear glass tube. After drying by vacuum centrifugation, the residue was reconstituted in $100 \mu \mathrm{L}$ of $50 \%$ internal standard solution, and $20 \mu \mathrm{L}$ of the dissolved solution was injected into the HPLC column.

\section{Ex vivo curcumin absorption using reverted rat gut sacs}

The ex vivo drug absorption study was performed as previous described. ${ }^{18}$ Male Sprague-Dawley rats $(210 \pm 10 \mathrm{~g}$ body weight) were sacrificed by intraperitoneal overdose using $1 \mathrm{~mL} / \mathrm{kg}$ urethane $(1 \mathrm{~g} / \mathrm{mL})$ and $\alpha$-chloralose $(0.1 \mathrm{~g} / \mathrm{mL})$. The entire small intestine was quickly removed and douched several times with normal saline $(0.9 \%, \mathrm{w} / \mathrm{v})$ at room temperature. The intestine was put into warm $\left(37^{\circ} \mathrm{C}\right)$ TC-199 medium ( $\mathrm{pH} 7.4)$ and separated into the duodenum, jejunum, and ileum. The gut was then gently reverted using a glass rod (2.5 $\mathrm{mm}$ diameter). The large gut sac was divided into small sacs of approximately $2 \mathrm{~cm}$ in length, then filled with TC-199 medium and tied with a silk suture.

Each of the small sacs was incubated in a conical flask containing $19.5 \mathrm{~mL}$ of TC-199 medium in a gentle shaking water bath at $37^{\circ} \mathrm{C}$. Next, $0.5 \mathrm{~mL}$ of curcumin $100 \mu \mathrm{g} / \mathrm{mL}$, LMw-NPC, and HMw-NPC were added into conical flasks. After incubating for one hour, each sac was cut open, and the serosal fluid and sac tissue collected into a clear tube; $1 \mathrm{~mL}$ of acetonitrile was added to the sac tissue, which was then homogenized and centrifuged at $6000 \mathrm{rpm}$ for 10 minutes.
A $50 \mu \mathrm{L}$ quantity of the sac supernatant and serosal fluid was added to $100 \mu \mathrm{L}$ of internal standard solution for protein precipitation and centrifuged at $13,000 \mathrm{rpm}$ for 10 minutes. The supernatants $(20 \mu \mathrm{L})$ were collected and analyzed by HPLC.

\section{Pharmacokinetics and statistics}

Pharmacokinetic values were calculated from each individual set of data using WinNonlin, standard edition, version 1.1 (Pharsight Corporation, Mountain View, CA) and the noncompartmental method. The relative oral bioavailability of curcumin was calculated according to the equation:

$$
\begin{aligned}
& \text { Relative } \mathrm{BA}(\%)= 100 \times\left[\left(\mathrm{AUC}_{\text {formulation }} / \text { dose }_{\text {formulation }}\right)\right. \\
&\left./\left(\mathrm{AUC}_{\text {curcumin }} / \text { dose }_{\text {curcumin }}\right)\right]
\end{aligned}
$$

The pharmacokinetic results are represented as the mean \pm standard error. The statistical analysis was performed by $t$-test (SPSS version 10.0, SPSS Inc, Chicago, IL) to compare different groups. The level of statistical significance was set at $P<0.05$.

\section{Results}

\section{Characteristics of LMw-NPC and $\mathrm{HMw}-\mathrm{NPC}$}

The properties of the LMw-NPC and HMw-NPC are summarized in Table 1. The zeta potential refers to the surface charge on the nanoparticles and the polydispersity index is the size distribution, indicating the degree of similarity between the nanoparticles, such that a smaller polydispersity index indicates that the particles are similar in size. Nanoparticles containing curcumin were prepared using a high-pressure emulsification-solvent evaporation procedure with PLGA as the encapsulating material. A negative zeta potential and smaller polydispersity index was obtained $(-12.2 \mathrm{mV}$ and 0.076 for $\mathrm{LMw}-\mathrm{NPC}$ versus $-14.1 \mathrm{mV}$ and 0.068 for HMw-NPC). The sizes of the LMw-NPC and HMw-NPC were $166 \pm 7.4 \mathrm{~nm}$ and $163 \pm 4.2 \mathrm{~nm}$, respectively. The encapsulation efficiency of both nanoformulations was nearly $45 \%$. According to the results, there was no statistically significant difference in particle characteristics between LMw-NPC and HMw-NPC. As in our previous studies, ${ }^{3,17}$ similar properties of nanoparticles were found in the different batches when complied with standard procedures to prepare these LMw-NPC. Therefore, our study results were confirmed to be replicable and were well validated. Figure 1 shows the TEM images of LMw-NPC and HMw-NPC. The morphology of the curcumin nanoparticles was either ellipsoid or spherical, 
Table I Effects of different molecular weights on nanoparticle characteristics

\begin{tabular}{lllll}
\hline & $\begin{array}{l}\text { Particle size } \\
(\mathbf{n m})\end{array}$ & $\begin{array}{l}\text { Polydispersity } \\
\text { index }\end{array}$ & $\begin{array}{l}\text { Zeta potential } \\
(\mathbf{m V})\end{array}$ & $\begin{array}{l}\text { Encapsulation } \\
\text { efficiency }(\%)\end{array}$ \\
\hline LMw-NPC & $166 \pm 7.4$ & $0.076 \pm 0.009$ & $-14.1 \pm 1.6$ & $46.1 \pm 3.7$ \\
HMw-NPC & $163 \pm 4.2$ & $0.068 \pm 0.012$ & $-12.2 \pm 1.0$ & $45.0 \pm 4.1$ \\
\hline
\end{tabular}

Note: Data expressed as mean \pm standard deviation $(n=3)$.

Abbreviations: LMw-NPC, curcumin encapsulated in low molecular weight PLGA; HMw-NPC, curcumin encapsulated in high molecular weight PLGA.

and the diameter results were similar to the data observed by dynamic light scattering (below $200 \mathrm{~nm}$ ) for both LMwNPC and HMw-NPC. Our TEM data are also consistent with those reported by Liang et al and Chang et al, ${ }^{19,20}$ with regard to the nanoparticles being a bright color and surrounded by a dark shadow. Our curcumin-loaded PLGA nanoparticles were well prepared and could be used in our subsequent pharmacokinetic study.

\section{Validation of HPLC analytic method}

The HPLC method was developed to investigate curcumin in rat plasma samples. Figure 2A-E shows the chromatograms of blank rat matrices, and Figure $2 \mathrm{~F}-\mathrm{J}$ shows the chromatograms of rat biosamples containing the internal standard and curcumin after administration of HMw-NPC. The retention times were approximately 6.5 and 22 minutes for the internal standard and curcumin, respectively, with no obvious interference peak in the blank chromatograms. These results suggest that the HPLC analytical conditions provided good separation and selectivity for curcumin and the internal standard in the experimental matrices. The validation methods were for linearity, limit of detection, limit of quantification, precision, accuracy, and extraction recovery. The calibration curves for plasma, feces, urine, serosal fluid, and sac tissue showed good linearity $\left(\mathrm{r}^{2}>0.995\right)$ over the range of $0.01-2.5 \mu \mathrm{g} / \mathrm{mL}$. The limits of detection and quantification for curcumin were determined to be $0.005 \mu \mathrm{g} / \mathrm{mL}$ and $0.01 \mu \mathrm{g} / \mathrm{mL}$, respectively. Intra-assay and interassay precision (\% RSD and accuracy [\% bias]) of curcumin measurements in each rat matrix were $\pm 15 \%$. Extraction of curcumin at low, medium, and high concentration in rat plasma, feces, urine, serosal fluid, and sac tissue was at least $90 \%$. These results indicate that the analytical and extraction methods used for curcumin and the internal standard were reliable for the following pharmacokinetic study.

\section{Bioavailability and pharmacokinetics}

Mean concentrations of curcumin in rat plasma at different times after oral administration of conventional curcumin $(1 \mathrm{mg} / \mathrm{kg})$ and LMw-NPC and HMw-NPC $(50 \mathrm{mg} / \mathrm{kg})$ are shown in Figure 3. Curcumin could be detected in plasma for 6 hours after oral ingestion of conventional curcumin and of LMw-NPC, but the retention time in rat plasma was prolonged to 8 hours for the HMw-NPC formulation. Table 2 shows significant increases in $\mathrm{C}_{\max }$ (from 0.028 for curcumin alone to 0.042 for $\mathrm{LMw}-\mathrm{NPC}$ to $0.057 \mu \mathrm{g} / \mathrm{mL}$ for HMw-NPC) and area under the concentration-time curve (AUC) per dose (from 0.0062 to 0.15 to 0.25 , respectively),
A

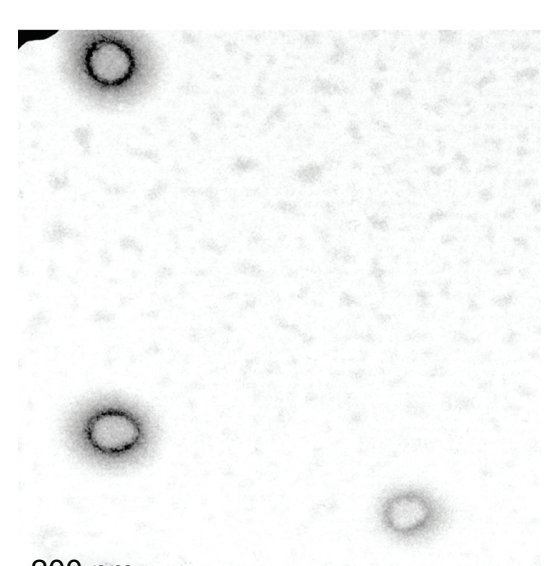

B

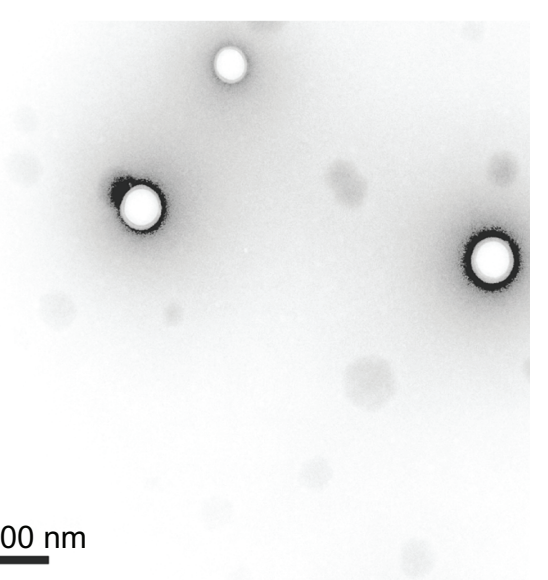

Figure I Morphology by transmission electron microscopy of curcumin nanoparticles. (A) LMw-NPC and (B) HMw-NPC.

Abbreviations: LMw-NPC, curcumin encapsulated in low molecular weight PLGA; HMw-NPC, curcumin encapsulated in high molecular weight PLGA. 

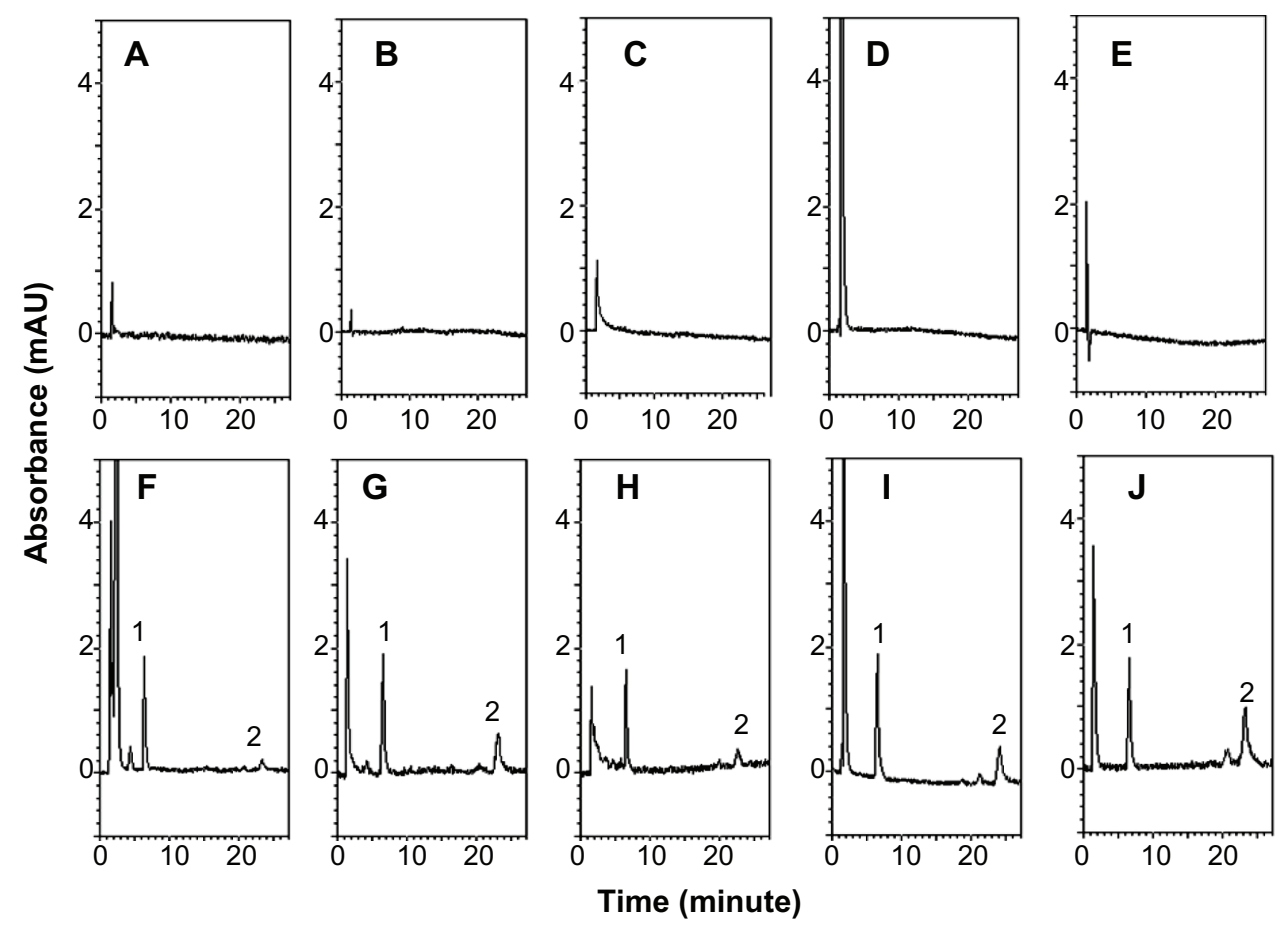

Figure 2 HPLC chromatograms of (A) blank plasma, (B) blank feces, (C) blank urine, (D) blank serosal fluid, (E) blank sac tissue, (F) plasma sample obtained from the jugular vein at 30 minutes after HMw-NPC administration $(50 \mathrm{mg} / \mathrm{kg}$, orally), (G) feces, and (H) urine samples collected by metabolic cages at I2-24 hours after HMw-NPC administration ( $50 \mathrm{mg} / \mathrm{kg}$, orally), (I) serosal fluid, and (J) sac tissue samples incubated HMw-NPC at I00 $\mu \mathrm{g} / \mathrm{mL}$ for 60 minutes. Peak I, 2-(4'-hydroxybenzeneazo)benzoic acid (as internal standard); peak 2, curcumin.

Abbreviation: HMw-NPC, curcumin encapsulated in high molecular weight PLGA.

indicating that the absorption of curcumin increased when it was encapsulated in a nanoformulation. In addition, the relative bioavailability of HMw-NPC was 40-fold and 1.67-fold higher than that of conventional curcumin and LMw-NPC, respectively (Table 2). This indicates that the HMw-PLGA nanoformulation achieved markedly better absorption of curcumin compared with LMw-PLGA. Furthermore, after oral administration of HMw-NPC, the $\mathrm{T}_{\text {max }}$ of curcumin was 15 minutes shorter than that of the other curcumin formulations (see Table 2). These results indicate that the relative bioavailability of curcumin was increased at a shorter $\mathrm{T}_{\max }$, indicating that $\mathrm{T}_{\max }$ was closely related to the bioavailability of curcumin.

Without nanoformulation, the accumulated percentage of unabsorbed curcumin in feces approached $90 \%$ and in urine was almost $0 \%$ (Figure 4), implying that there was low absorption of curcumin in the gastrointestinal tract. PLGA encapsulation reduced the amount of curcumin in feces (from $90 \%$ for curcumin alone to $40 \%$ for LMw-PLG to $20 \%$ for HMw-PLGA) and increased the amount of curcumin in urine (from near $0 \%$ to $0.07 \%$ to $0.16 \%$ ), respectively, indicating that the absorption of curcumin was enhanced after PLGA formulation. These excretion results confirmed the pharmacokinetic parameters of the oral study, ie, the absorption of curcumin was significantly increased by both LMw-PLGA and HMw-PLGA formulation.

\section{Ex vivo absorption}

To understand better the relationship between relative bioavailability and $\mathrm{T}_{\max }$ for different molecular weights of PLGA-encapsulated curcumin and to explore the possible reasons for this, an ex vivo absorption study was undertaken. The concentration of curcumin absorbed from the incubation medium into the sac tissue and then into the serosal fluid is shown in Figure 5. When samples of the reverted sac of rat small intestine were incubated with conventional curcumin, LMw-NPC, and HMw-NPC, the amount of curcumin absorbed from the curcumin nanoformulation was significantly increased in the duodenum and the ileum, but was not significantly different in the jejunum. The curcumin content was highest in the serosal fluid of the duodenum in the HMw-NPC group, followed by the LMw-NPC and conventional curcumin groups. The curcumin concentration from HMw-NPC in the serosal fluid of the duodenum was 5.12-fold and 2.66-fold higher than from LMw-NPC and conventional curcumin, respectively (Figure 5A).

The absorption of curcumin, as determined by the sum of the amounts of curcumin in the serosal fluid, sac tissue, 


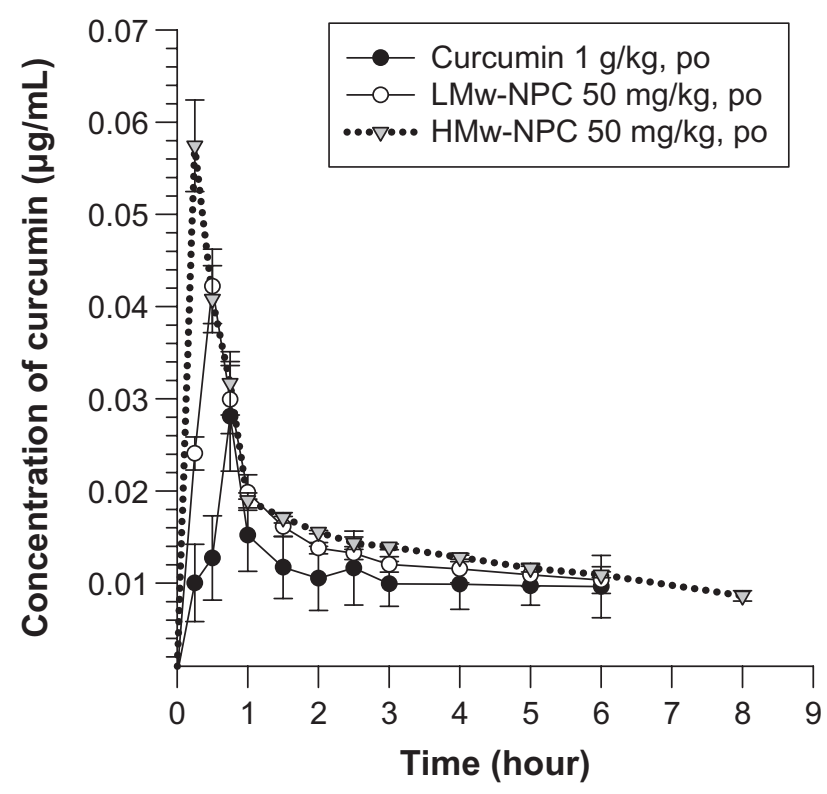

Figure 3 Concentration-time curve of curcumin in rat plasma after oral administration of curcumin at I g/ $/ \mathrm{kg}$, and LMw-NPC and HMw-NPC at $50 \mathrm{mg} / \mathrm{kg}$ (mean \pm standard error, $n=4$ ).

Notes: • curcumin; o, LMw-NPC; $\boldsymbol{\nabla}, \mathrm{HMw}-\mathrm{NPC}$.

Abbreviations: LMw-NPC, curcumin encapsulated in low molecular weight PLGA; $\mathrm{HMw}-\mathrm{NPC}$, curcumin encapsulated in high molecular weight PLGA.

and regions of the rat gut after incubation with curcumin and its formulations, is shown in Table 3. It can be seen that the amount of curcumin in the duodenum and ileum was significantly increased by curcumin encapsulated with PLGA. The HMw-PLGA formulation noticeably enhanced the absorption of curcumin in the duodenum compared with LMw-PLGA (from $0.63 \mu \mathrm{g}$ to $0.89 \mu \mathrm{g}$ ) and with conventional curcumin (from $0.47 \mu \mathrm{g}$ to $0.89 \mu \mathrm{g}$ ). The ex vivo curcumin absorption results support the findings of the oral study, ie, that curcumin formulated with PLGA increased the absorption of curcumin in the intestine. Overall, HMwPLGA enabled considerably more curcumin absorption than LMw-PLGA.

Table 2 Pharmacokinetic parameters of curcumin in rat plasma after oral administration

\begin{tabular}{|c|c|c|c|}
\hline \multirow[t]{2}{*}{ Parameter } & Curcumin & LMw-NPC & HMw-NPC \\
\hline & $\mathrm{I} \mathrm{g/kg}$ & $50 \mathrm{mg} / \mathrm{kg}$ & $50 \mathrm{mg} / \mathrm{kg}$ \\
\hline $\mathrm{T}_{\max }(\min )$ & 45 & 30 & 15 \\
\hline$C_{\text {max }}(\mu g / m L)$ & $0.028 \pm 0.01$ & $0.042 \pm 0.005^{a}$ & $0.057 \pm 0.005^{\mathrm{a}, \mathrm{b}}$ \\
\hline AUC $(\min \mu g / m L)$ & $6.23 \pm 0.86$ & $7.52 \pm 0.63^{\mathrm{a}}$ & $12.5 \pm 0.82^{\mathrm{a}, \mathrm{b}}$ \\
\hline AUC/dose & $0.0062 \pm 0.003$ & $0.15 \pm 0.009^{a}$ & $0.25 \pm 0.018^{\mathrm{a}, \mathrm{b}}$ \\
\hline Relative BA & I & 24 & 40 \\
\hline
\end{tabular}

Notes: Data expressed as mean \pm standard error of the mean $(n=4)$. ${ }^{a} p<0.05$, significantly different from curcumin; ${ }^{b} P<0.05$, significantly different from LMw-NPC. Abbreviations: $C_{\max }$, the maximum plasma concentration; $T_{\max }$, time to $C_{\max }$; AUC, area under the concentration-time curve; BA, bioavailability; LMw-NPC, curcumin encapsulated in low molecular weight PLGA; HMw-NPC, curcumin encapsulated in high molecular weight PLGA.
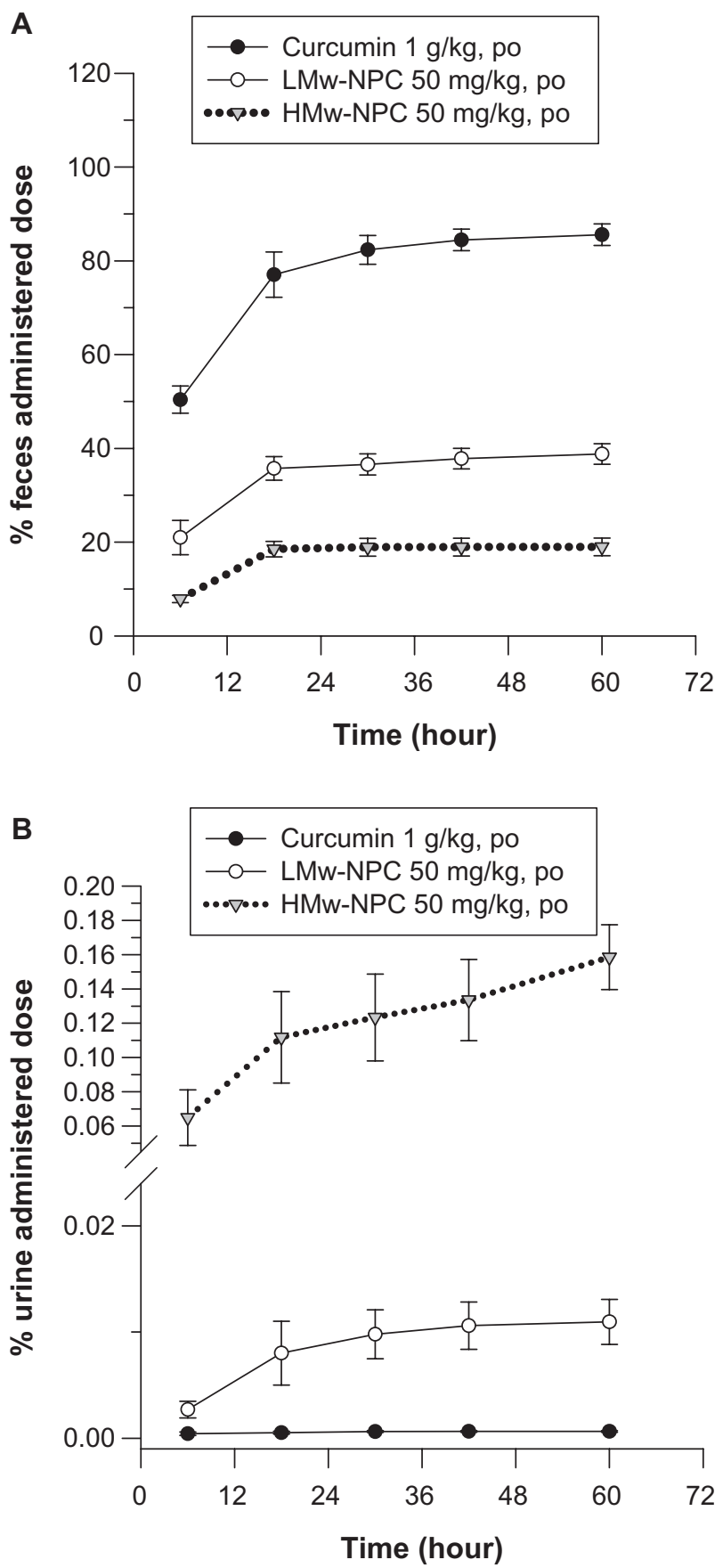

Figure 4 Cumulative excretion of curcumin in (A) feces and (B) urine after oral administration of curcumin at $\mathrm{I} g / \mathrm{kg}$, and LMw-NPC and HMw-NPC at $50 \mathrm{mg} / \mathrm{kg}$ (mean \pm standard error, $n=4$ ).

Notes: • curcumin; o, LMw-NPC; $\boldsymbol{\nabla}, \mathrm{HMw}-\mathrm{NPC}$

Abbreviations: LMw-NPC, curcumin encapsulated in low molecular weight PLGA; $\mathrm{HMw}-\mathrm{NPC}$, curcumin encapsulated in high molecular weight PLGA

\section{Discussion}

Nanoformulation technology has been extensively utilized in foods and nutritional supplements to increase the oral bioavailability of active compounds, which may be beneficial for human health. However, there has been little investigation of how the materials used in the nanoformulation process affect 

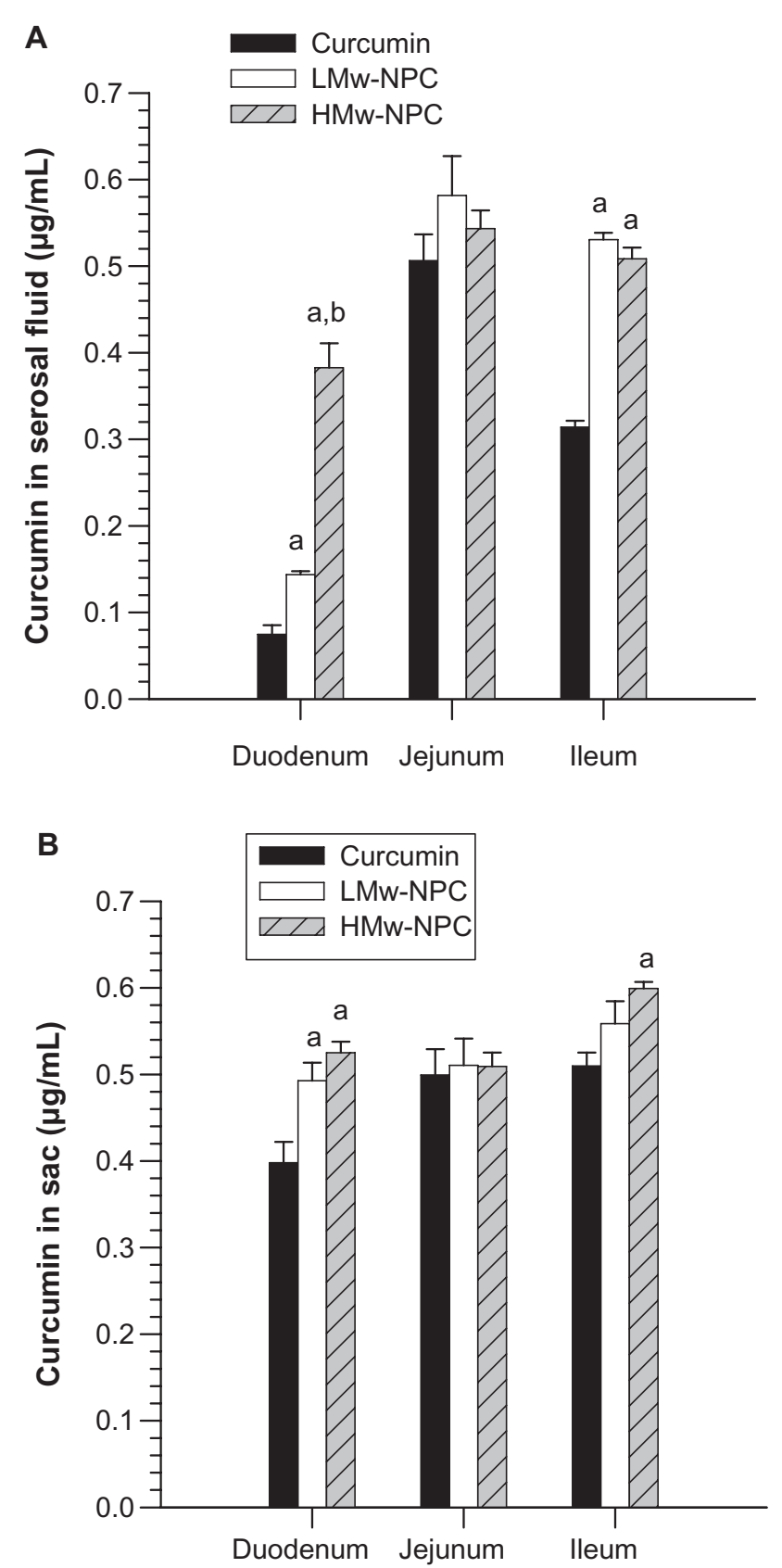

Figure 5 Concentration of curcumin in (A) serosal fluid and (B) sac tissue of different small intestine region after incubation of curcumin, and LMw-NPC and $\mathrm{HMw}-\mathrm{NPC}$ at $100 \mu \mathrm{g} / \mathrm{mL}$ for one hour.

Notes: ${ }^{a} P<0.05$, significantly different from curcumin; ${ }^{b} P<0.05$, significantly different from LMw-NPC.

Abbreviations: LMw-NPC, curcumin encapsulated in low molecular weight PLGA; HMw-NPC, curcumin encapsulated in high molecular weight PLGA.

the oral pharmacokinetics of a product. This study found that changing the molecular weight of PLGA changes the pharmacokinetic parameters of conventional curcumin after oral administration. The present study also investigated the possible mechanism for this difference. The HPLC method was optimized and validated to obtain reliable pharmacokinetic data for curcumin ${ }^{21}$ (Figure 2).
Table 3 Amount of curcumin in sections of the small intestine after incubation of curcumin, LMw-NPC, and HMw-NPC for 60 minutes

\begin{tabular}{lccc}
\hline $\begin{array}{l}\text { Subdivision of } \\
\text { small intestine }\end{array}$ & \multicolumn{3}{c}{ Curcumin amount after incubation $(\mu \mathrm{g})$} \\
\cline { 2 - 4 } & Curcumin & LMw-NPC & HMw-NPC \\
\hline Serosal fluid + sac tissue & & \\
Duodenum & $0.47 \pm 0.04$ & $0.63 \pm 0.04^{\mathrm{a}}$ & $0.89 \pm 0.07^{\mathrm{a}, \mathrm{b}}$ \\
Jejunum & $1.0 \mathrm{I} \pm 0.05$ & $1.12 \pm 0.06$ & $\mathrm{I} .1 \mathrm{I} \pm 0.0 \mathrm{I}$ \\
lleum & $0.85 \pm 0.02$ & $1.02 \pm 0.02^{\mathrm{a}}$ & $\mathrm{I} . \mathrm{II} \pm 0.04^{\mathrm{a}}$ \\
\hline
\end{tabular}

Notes: Data expressed as mean \pm standard error $(n=4)$. a $P<0.05$, significantly different from curcumin; ${ }^{b} P<0.05$, significantly different from LMw-NPC.

Abbreviations: LMw-NPC, curcumin encapsulated in low molecular weight PLGA; $\mathrm{HMw}-\mathrm{NPC}$, curcumin encapsulated in high molecular weight PLGA.

The results of the animal study showed that the bioavailability of curcumin was significantly increased by nanoformulation when using low or high molecular weight PLGA. The bioavailability of a compound in the body can be enhanced by decreasing its metabolism in the body or by increasing the absorption of the compound in the gastrointestinal tract. ${ }^{22}$ Transit time in the stomach and intestines is a major factor affecting absorption, and a longer intestinal transit time will generally enhance the absorption of a substance. ${ }^{23}$ In our study, the transit time of curcumin in the gastrointestinal tract was investigated to determine the effect of absorption. We undertook an excretion study using a metabolic cage, and the fecal excretion data (Figure 4A) showed that the time approaching the fastest rate and maximum accumulated amount of curcumin in feces was 18 hours, after which a plateau was maintained for 18-60 hours in all groups. This indicates that the time for curcumin to transit the rat gastrointestinal tract is independent of whether curcumin is formulated with or without LMw-PLGA or HMw-PLGA. This finding suggests that increased bioavailability is not due to the prolonged transit time of curcumin through the gastrointestinal tract as a result of formulation with PLGA. On the other hand, the hydrophilic characteristics of a compound will affect its absorption in the body. Merisko-Liversidge et al reported that nanoformulations increase the surface area and surface interactions of a compound compared with conventional forms, and that such formulations also increase the rate at which compounds are dissolved in the gastrointestinal tract, thereby improving the absorption of water-insoluble compounds. ${ }^{9}$ Therefore, increased bioavailability of curcumin in the body may be attributable to faster dissolution after administration via a nanoformulation. We found that the $\mathrm{C}_{\max }, \mathrm{AUC}$, and AUC per dose for the HMwNPC group were significantly higher than for the LMw-NPC group (Table 2), even though the two groups had similar nanoparticle characteristics (Table 1). This may be because 
HMw-PLGA was more resistant than LMw-PLGA to the very acidic environment of gastric juice, which could reduce absorption by damaging the nanoparticle structure. ${ }^{24}$

It is noteworthy that different $\mathrm{T}_{\max }$ values were observed in the different curcumin formulation groups (Table 2). Denoting the time to maximum concentration of a drug in plasma, $\mathrm{T}_{\max }$ can be used as an indicator of the drug absorption rate, with smaller values indicating more rapid absorption. ${ }^{25}$ According to our findings, the $\mathrm{T}_{\max }$ of conventional curcumin, LMw-NPC, and HMw-NPC was 45, 30, and 15 minutes, respectively. This indicates that absorption of HMw-NPC was faster than that of LMw-NPC, followed by conventional curcumin. A previous study found that oral absorption of a drug is influenced by particle size, which affects the dissolution rate. ${ }^{26}$ However, the current study found no statistically significant difference in particle size or any other particle characteristics between HMw-NPC and LMw-NPC (Table 1). Thus, particle size and other characteristics might not be responsible for the different bioavailability and absorption results for HMw-NPC and LMw-NPC. We hypothesized that the observed differences in bioavailability and $\mathrm{T}_{\max }$ may be due to different absorption rates in different portions of the gastrointestinal tract. To verify this hypothesis, we investigated ex vivo curcumin absorption from a portion of the small intestine.

It is known that the small intestine, composed of the duodenum, jejunum, and ileum, is the main site for digestion and absorption of water, nutrients, and electrolytes after a substance has been ingested orally. ${ }^{27}$ Our ex vivo absorption study demonstrated that conventional curcumin was absorbed mostly in the jejunum (Table 3 ). When encapsulated with PLGA, the amount of curcumin absorbed in the duodenum and ileum increased significantly, especially for HMw-NPC in the duodenum, but there was no significant difference in the rate of curcumin absorption in the jejunum with or without PLGA nanoformulation (Table 3).

Although DeSesso et al have reported that the bulk of absorption takes place in the duodenum and proximal half of the jejunum, ${ }^{23}$ our results indicate that the jejunum is the main area for absorption of curcumin. Encapsulating curcumin in a PLGA formulation will increase the amount of curcumin that can be absorbed in the duodenum, and even more so when curcumin is encapsulated with larger molecular weight PLGA. Therefore, increasing the absorption rate of curcumin in the duodenum may play an important role in increasing the relative bioavailability of curcumin formulated with PLGA. Rapid absorption of HMw-NPC in the duodenum would have shortened the $\mathrm{T}_{\max }$ when any of our study formulations were ingested. Previous research has shown that the duodenum is the main absorption site in the intestine. ${ }^{23}$ Our results demonstrate that the bioavailability of a compound that cannot be effectively absorbed can be increased by high molecular weight PLGA, which is a significant and useful finding for nanoformulation. Possible transport mechanisms for this phenomenon will be investigated in a further study.

In conclusion, this study demonstrates that formulation with PLGA could enhance the bioavailability of a waterinsoluble polyphenol by increasing the absorption rate in the duodenum. In addition, different molecular weights of PLGA affect the pharmacokinetics of curcumin, which are closely related to the rate of absorption in the duodenum. These findings provide meaningful information for PLGA nanoformulations intended to increase the bioavailability of hydrophobic compounds in the design of drug delivery systems, and can be utilized in further preclinical and clinical research.

\section{Acknowledgments}

Funding for this study was provided in part by research grants from the National Science Council, Taiwan (NSC992113-M-010-001-MY3, NSC99-2628-B-010-008-MY3), Taipei City Hospital (TCH99001-62-007), and Technology Development Program for Academia, Ministry of Economic Affairs, Taiwan (99-EC-17-A-17-S1-152).

\section{Disclosure}

The authors report no conflicts of interest in this work.

\section{References}

1. Quideau S, Deffieux D, Douat-Casassus C, et al. Plant polyphenols: chemical properties, biological activities, and synthesis. Angew Chem Int Ed Engl. 2011;50(3):586-621.

2. Leonarduzzi G, Testa G, Sottero B, et al. Design and development of nanovehicle-based delivery systems for preventive or therapeutic supplementation with flavonoids. Curr Med Chem. 2010;17(1): 74-95.

3. Tsai YM, Jan WC, Chien CF, et al. Optimised nano-formulation on the bioavailability of hydrophobic polyphenol, curcumin, in freely-moving rats. Food Chem. 2011;127(3):918-925.

4. Johnson JJ, Nihal M, Siddiqui IA, et al. Enhancing the bioavailability of resveratrol by combining it with piperine. Mol Nutr Food Res. 2011 55(8):1169-1176.

5. Smith AJ, Kavuru P, Wojtas L, et al. Cocrystals of quercetin with improved solubility and oral bioavailability. Mol Pharm. 2011;8(5):1867-1876.

6. Zhang Z, Huang Y, Gao F, et al. A self-assembled nanodelivery system enhances the oral bioavailability of daidzein: in vitro characteristics and in vivo performance. Nanomedicine (Lond). 2011;6(8):1365-1379.

7. Parveen R, Baboota S, Ali J, et al. Oil based nanocarrier for improved oral delivery of silymarin: in vitro and in vivo studies. Int J Pharm. 2011; 413(1-2):245-453.

8. Yu JN, Zhu Y, Wang L, et al. Enhancement of oral bioavailability of the poorly water-soluble drug silybin by sodium cholate/phospholipid-mixed micelles. Acta Pharmacol Sin. 2010;31(6):759-764. 
9. Merisko-Liversidge EM, Liversidge GG. Drug nanoparticles: formulating poorly water-soluble compounds. Toxicol Pathol. 2008; 36(1):43-48.

10. des Rieux A, Fievez V, Garinot M, et al. Nanoparticles as potential oral delivery systems of proteins and vaccines: a mechanistic approach. J Control Release. 2006;116(1):1-27.

11. ParkTG. Degradation of poly(lactic-co-glycolic acid) microspheres: effect of copolymer composition. Biomaterials. 1995;16(15):1123-1130.

12. Yallapu MM, Ebeling MC, Chauhan N, et al. Interaction of curcumin nanoformulations with human plasma proteins and erythrocytes. Int J Nanomedicine. 2011;6:2779-2790.

13. Sonaje K, Italia JL, Sharma G, et al. Development of biodegradable nanoparticles for oral delivery of ellagic acid and evaluation of their antioxidant efficacy against cyclosporine A-induced nephrotoxicity in rats. Pharm Res. 2007;24(5):899-908.

14. Ghosh A, Mandal AK, Sarkar S, et al. Nanoencapsulation of quercetin enhances its dietary efficacy in combating arsenic-induced oxidative damage in liver and brain of rats. Life Sci. 2009;84(3-4):75-80.

15. Yallapu MM, Gupta BK, Jaggi M, et al. Fabrication of curcumin encapsulated PLGA nanoparticles for improved therapeutic effects in metastatic cancer cells. J Colloid Interface Sci. 2010;351(1):19-29.

16. Xie X, Tao Q, Zou Y, et al. PLGA nanoparticles improve the oral bioavailability of curcumin in rats: characterizations and mechanisms. $J$ Agric Food Chem. 2011;59(17):9280-9289.

17. Tsai YM, Chien CF, Lin LC, et al. Curcumin and its nano-formulation: the kinetics of tissue distribution and blood-brain barrier penetration. Int J Pharm. 2011;416(1):331-338.

18. Barthe L, Woodley J, Houin G. Gastrointestinal absorption of drugs: methods and studies. Fundam Clin Pharmacol. 1999;13(2):154-168.
19. Liang HF, Yang TF, Huang CT, et al. Preparation of nanoparticles composed of poly(gamma-glutamic acid)-poly(lactide) block copolymers and evaluation of their uptake by HepG2 cells. J Control Release. 2005;105(3):213-225.

20. Chang $\mathrm{CH}$, Huang WY, Lai $\mathrm{CH}$, et al. Development of novel nanoparticles shelled with heparin for berberine delivery to treat Helicobacter pylori. Acta Biomater. 2011;7(2):593-603.

21. Shah VP, Midha KK, Dighe S, et al. Analytical methods validation: bioavailability, bioequivalence and pharmacokinetic studies. Conference report. Eur J Drug Metab Pharmacokinet. 1991;16(4): 249-255.

22. Fasinu P, Pillay V, Ndesendo VM, et al. Diverse approaches for the enhancement of oral drug bioavailability. Biopharm Drug Dispos. 2011; 32(4):185-209.

23. DeSesso JM, Jacobson CF. Anatomical and physiological parameters affecting gastrointestinal absorption in humans and rats. Food Chem Toxicol. 2001;39(3):209-228.

24. Zolnik BS, Burgess DJ. Effect of acidic pH on PLGA microsphere degradation and release. J Control Release. 2007;122(3):338-344.

25. Shargel L, Wu-Pong S, Yu A, et al. Bioavailability and bioequivalence. In: Applied Biopharmaceutics and Pharmacokinetics, 5th ed. Boston, MA: McGraw-Hill Companies; 2004.

26. Xia D, Cui F, Piao H, et al. Effect of crystal size on the in vitro dissolution and oral absorption of nitrendipine in rats. Pharm Res. 2010; 27(9):1965-1976.

27. Kato A, Romero MF. Regulation of electroneutral $\mathrm{NaCl}$ absorption by the small intestine. Annu Rev Physiol. 2011;73:261-281.
International Journal of Nanomedicine

\section{Publish your work in this journal}

The International Journal of Nanomedicine is an international, peerreviewed journal focusing on the application of nanotechnology in diagnostics, therapeutics, and drug delivery systems throughout the biomedical field. This journal is indexed on PubMed Central, MedLine, CAS, SciSearch $\AA$, Current Contents ${ } /$ Clinical Medicine,

\section{Dovepress}

Journal Citation Reports/Science Edition, EMBase, Scopus and the Elsevier Bibliographic databases. The manuscript management system is completely online and includes a very quick and fair peer-review system, which is all easy to use. Visit http://www.dovepress.com/ testimonials.php to read real quotes from published authors. 